EPJ Web of Conferences 81, 03008 (2014)

DOI: $10.1051 /$ epjconf/ 20148103008

(C) Owned by the authors, published by EDP Sciences, 2014

\title{
$B$ meson decays in leptons: powerful probes of new physics
}

\author{
Marcello Rotondo ${ }^{1, a}$ \\ for the BaBar Collaboration \\ ${ }^{1}$ I.N.F.N Sezione di Padova, Via Marzolo 8, 35131 Padova - Italy
}

\begin{abstract}
We review some recent measurements of $B$ meson decays that involve leptons in the final states and that are sensitive to physics beyond the Standard Model, such as the electroweak penguin decays $B \rightarrow X_{s} \ell^{+} \ell^{-}$, the Lepton Number Violating process $B \rightarrow$ $X \ell^{ \pm} \ell^{\prime \pm}$ and the tree-level dominated decay with $\tau$ leptons: $B \rightarrow \tau v_{\tau}$ and $B \rightarrow D^{(*)} \tau v_{\tau}$.
\end{abstract}

\section{Introduction}

The BaBar experiment has collected about $470 \cdot 10^{6}$ couple of $B \bar{B}$ events from the $\Upsilon(4 S)$ decays. This huge sample allowed to perform stringent studies on $B$ meson properties and successfully confirmed the Standard Model (SM) predictions in the flavour sector. Although no relevant deviations from the SM have yet been found with the current statistics by BaBar and Belle, it is of paramount importance to continue to search for New Physics (NP) sign through indirect virtual contributions in the $B$ decays. Here we present some recent result from BaBar on $B$ meson decays in channels with leptons in the final state. All the results presented are based on the full available dataset.

\section{Inclusive $B \rightarrow X_{s} \ell^{+} \ell^{-}$decays}

The process $b \rightarrow s \ell^{+} \ell^{-}$, where $b$ is a bottom quark, $s$ is a strange quark, and $\ell^{+} \ell^{-}$is a $e^{+} e^{-}$or $\mu^{+} \mu$ - pair, is a FCNC process forbidden at lowest order in the Standard Model but is allowed via EW penguin and W-box diagrams. The amplitude for this decays is expressed in terms of perturbatively calculable effective Wilson coefficients. The non-SM contributions can modify the Wilson coefficients from the SM expectations. The exclusive channels $B \rightarrow K \ell^{+} \ell^{-}$and $B \rightarrow K^{*} \ell^{+} \ell^{-}$are currently extensively studied at B-Factories and LHCb. The study of the inclusive channel, where one does not look in a specific final state, is complementary to the exclusive studies.

Here we report a recent BaBar measurement [2] of the $B \rightarrow X_{s} \ell^{+} \ell^{-}$decay, using a sum over exclusive final states, which provides a basis for an extrapolation to the fully inclusive rate. We measure the total Branching Fraction $(B F)$ as well as partial $B F$ in five bins of $q^{2} \equiv m_{\ell^{+} \ell^{-}}^{2}$. The $X_{s}$ states are reconstructed in 10 separate $X_{s}$ hadronic final states $\left(K^{+}, K^{+} \pi^{0}, K^{+} \pi^{-} \pi^{0}, K^{+} \pi^{-} \pi^{+}, K_{s}^{0}\right.$, $K_{s}^{0} \pi^{0}, K_{s}^{0} \pi^{+}, K_{s}^{0} \pi^{+} \pi^{0}$ and $\left.K_{s}^{0} \pi^{+} \pi^{-}\right)$, combining these with $e^{+} e^{-}$and $\mu^{+} \mu^{-}$pairs. We limit the number of pions in the final state to two and require $m_{X_{s}}<1.8 \mathrm{GeV} / c^{2}$ since the expected $S / N$ ratio decrease very rapidly with the mass and the $X_{S}$ pion multiplicity. The reconstructed states, taking into account

ae-mail: marcello.rotondo@pd.infn.it 
the presence of the $K_{L}$ and the $K_{s} \rightarrow \pi^{0} \pi^{0}$, represent about $70 \%$ of the total inclusive rate. We suppress the $e^{+} e^{-} \rightarrow q \bar{q}$ events (where $q=u, d, s$, or $c$ quark) and $B \bar{B}$ combinatoric background using Boosted Decision Trees (BDTs) exploiting many kinematical and topological quantities. In each $q^{2}$ bin, we extract the signal yield with a 2-D maximum likelihood fit using the mass of the $B$ meson candidate and a likelihood ratio based on the BDTs used to suppress the $B \bar{B}$ backgrounds. From the extracted signal yields and the efficiencies, we compute the partial $B F \mathrm{~s}$ in each $q^{2}$ bin. The measured partial $B F \mathrm{~s}$ in the various $q^{2}$ bins analysed is reported in Fig. 1. The lepton-flavour averaged result for the

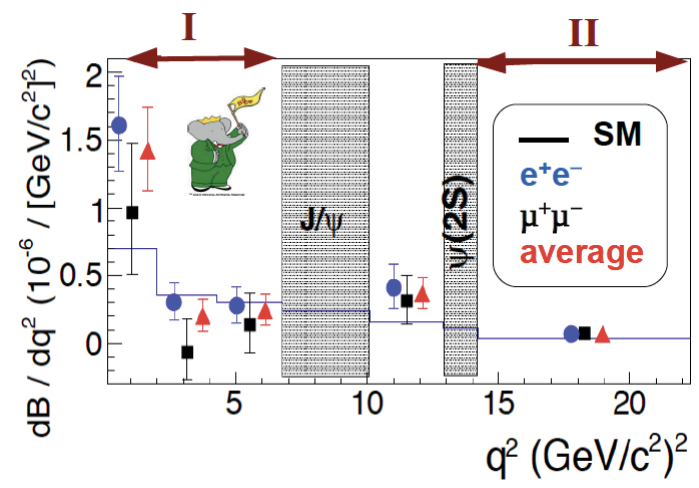

Figure 1. Differential $B F$ as a function of $q^{2}$ for $e^{+} e^{-}$, $\mu^{+} \mu^{-}$and lepton-flavour averaged final states. The histogram shows the SM expectation which is affected by an uncertainty of $10 \%-30 \%$ depending on the $q^{2}$ regions. The shaded boxes denote the vetoed regions to remove the copious production of $B \rightarrow X_{s} J / \psi$ and $B \rightarrow X_{s} \psi^{\prime}$, where $J / \psi\left(\psi^{\prime}\right) \rightarrow \ell^{+} \ell^{-}$. The region $\mathbf{I}$ and $\mathbf{I I}$ are defined respectively as $1<q^{2}<6 \mathrm{GeV}^{2} / c^{4}$ and $q^{2}>14.4 \mathrm{GeV}^{2} / c^{4}$.

range $1<q^{2}<6 \mathrm{GeV}^{2} / c^{4}$ ( I ) is $B F=\left(1.60_{-0.39}^{+0.44}{ }_{-0.13}^{+0.17} \pm 0.18\right) \cdot 10^{-6}$ and for $q^{2}>14.4 \mathrm{GeV}^{2} / c^{4}$ (III) is $B F=\left(0.57_{-0.15}^{+0.16}{ }_{-0.02}^{0.03} \pm 0.00\right) \cdot 10^{-6}$, where the uncertainties are statistical, systematics and due to the model-dependent extrapolation to the full rates. The measured rate is in very in good agreement with the SM predictions in region $\mathbf{I}\left(B F\left(B \rightarrow X_{s} \mu^{+} \mu^{-}\right)=(1.59 \pm 0.11) \cdot 10^{-6}\right.$ [3] $)$, and it is within $2 \sigma$ with the prediction in the region II $\left(B F\left(B \rightarrow X_{s} \mu^{+} \mu^{-}\right)=(0.24 \pm 0.07) \cdot 10^{-6}\right.$ [3] $)$. It is interesting to note that the $e^{+} e^{-}$results are systematically higher than the $\mu^{+} \mu^{-}$case, but they are compatible within the experimental uncertainties. It should be noted here that the explanation of the LHCb observation of the anomaly in one of the observables [4], as suggested in [5] would result in a decrease of the inclusive $B F$ of up to about $25 \%$ in both the region I and II and this is not compatible with our result.

\section{Search for $B \rightarrow X \ell^{ \pm} \ell^{\prime \pm}$}

The neutrino oscillation suggests that lepton number may not be conserved, moreover the leptonnumber violation (LNV) is a necessary condition for leptogenesis as an explanation of the baryon asymmetry of the Universe [7]. Many models beyond the SM predict the LNV in $B$ meson decays with a rate that could be accessible with the present data available [6]. CLEO performed [8] for the first time searches of LNV processes in the $B$ meson decays like $B^{+} \rightarrow X \ell^{+} \ell^{\prime}$, where $X$ is a charged particle or resonance and $\ell / \ell^{\prime}=e$ or $\mu$. Recently LHCb [11], BaBar [10] and Belle [9] reported search for these processes using much higher statistics.

Here we report the recent $\mathrm{BaBar}$ search [12] for $B^{+} \rightarrow X \ell^{+} \ell^{\prime+}$ with $X=K^{-}, \pi^{-} \rho^{-}, K^{*-}$ or $D^{-}$. The huge background is suppressed with a BDT that uses many event-shape and signal kinematic discriminant variables. The signal is extracted from a 3-D unbinned maximum likelihood fit to the BDT output, the $B$ candidate mass, and $\Delta E=E_{B}^{*}-E_{c . m}$, where $E_{B}^{*}$ is the energy of the $B$ candidate in the c.m. frame. We find no significant signal yields and place upper limits on the $B F$ in the range $(1.5-26) \cdot 10^{-7}$. These limits are in many cases more stringent than previous best measurements. In Fig. 2 we report a summary of the existing upper limits on a large set of studies $B$-meson LNV processes. 
MESON 2014 $-13^{\text {th }}$ International Workshop on Production, Properties and Interaction of Mesons

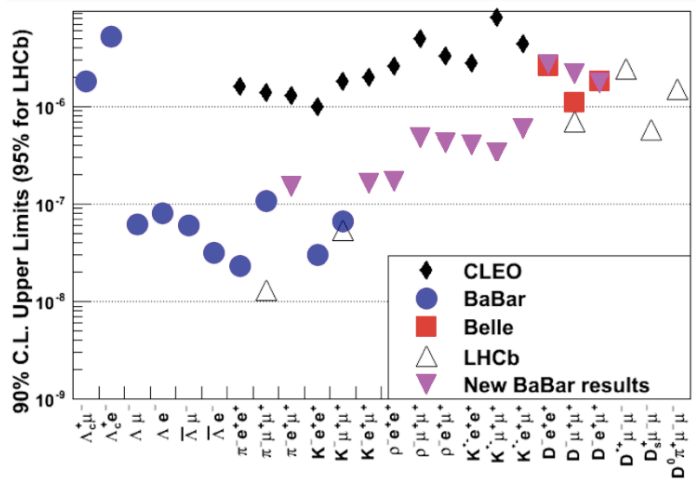

Figure 2. The upper limits on various $B$ meson LNV processes obtained by CLEO, Belle, BaBar and LHCb. The most recent upper limits ( $90 \%$ C.L.) on the $B F$ of various $B^{+} \rightarrow X \ell^{+} \ell^{\prime+}$ processes obtained by BaBar [12] are in the range $1.2 \cdot 10^{-7}-26 \cdot 20^{-7}$ and they are indicated with the filled triangles. For modes with $\rho, \pi$ and $K$ the limits are an order of magnitues more stringent than previous best results [8]. The upper limit for the $B \rightarrow D \ell^{+} \ell^{\prime+}$ are compatible wih those reported by Belle in Ref. [9].

\section{$4 B \rightarrow \tau v_{\tau}$ and $B \rightarrow D^{*} \tau v_{\tau}$}

The FCNC processes are powerful tools to search for physics beyond the SM, but tree level dominated processes are good as well to constraint important kind of NP. Many extended versions of the SM require changes in the Higgs sector, with the prediction of many Higgs bosons, both neutral and charged. A prototype of these models is the widely studies 2 Higgs-Doublet-Model of type-II, that predicts the existence of a charged Higgs $H^{+}$. A virtual $H^{+}$can replace the $W^{+}$in the leptonic $B \rightarrow \tau v$ and semileptonic $B \rightarrow D^{*} \tau v_{\tau}$ decays, which results in a significant enhancement and suppression of the predicted SM rates. These decays with $\tau$ in the final states are challenging to identify experimentally, since they include multiple neutrinos in the final state: one $v_{\tau}$ from the $W$ and one or two more from the $\tau$ decay. At the B-Factories these decays can be reconstructed exploiting the fact that only a $B$ meson pair is produced from the $e^{+} e^{-} \rightarrow \Upsilon(4 S) \rightarrow B \bar{B}$ process. It is possible to reconstruct one of the $B$ mesons $\left(B_{\text {tag }}\right)$ in a large number of decays and identify the signal decays $\left(B_{\text {sig }}\right)$ from the rest of the event.

The BaBar measurement [13] uses the hadronic tag where one of the $B$ meson of the $\Upsilon(4 S)$ decay is reconstructed in a large set of hadronic $B^{+} \rightarrow D^{(*) 0} X^{+}$decays or $B^{+} \rightarrow J / \psi X^{+}$decays (where $X^{+}$denotes a system of hadrons with charge +1 composed of combinations of $\pi^{+}, \pi^{0}, K^{ \pm}$and $K_{s}^{0}$, and the $D^{(*) 0}$ is reconstructed in many different decay channels to maximize the efficiency). Once the $B_{\text {tag }}$ is reconstructed, the rest of the event is due to the decay products of the $\tau$ that comes from the $B_{\text {sig }}$ decays. We reconstruct the $\tau$ in both leptonic and hadronic modes. The most significant discriminating variable is $E_{\text {extra }}$ defined as the sum of the energies of the neutral clusters not associated with the $B_{t a g}$ or with the products of the signal $\tau$. Signal events tend to peak at low $E_{\text {extra }}$, instead background event, which contain additional neutral clusters, tend to be distribute at higher values. We fit $N_{\text {sig }}=62.1 \pm 17.3$ signal events corresponding to $B F(B \rightarrow \tau v)=\left(1.83_{-0.49}^{+0.53}(\right.$ stat $) \pm 0.24($ syst $\left.)\right) \cdot 10^{-4}$, excluding the null hypothesis by $3.8 \sigma$. Our measurement is compatible with other measurements [14] and exceeds the prediction of the SM determination by $2.4 \sigma$ and $1.6 \sigma$ according to the used value of $\left|V_{u b}\right|$, exclusive or inclusive respectively. The $\mathrm{BaBar}$ result is marginally compatible with the most recent one obtained by Belle [15] obtained with an improved hadronic $B$ tag technique. The Belle result is perfectly compatible with the SM expectation. Despite the not clear situation on the $B \rightarrow \tau v$ decays, the measured $B F$ put not trivial constraints on $\tan \beta / m_{H^{+}}$for the $2 \mathrm{HDM}$ as shown in Fig. 3 (right).

BaBar published an interesting result [16] on the semileptonic $B \rightarrow D^{(*)} \tau v$ decays using the hadronic tag. The signal signature of the $B \rightarrow D^{(*)} \tau v$ decays are represented by a large value of missing mass, determined from the kinematic of the reconstructed $B_{\text {tag }}$, the signal $D^{(*)}$ and the lepton ( $e$ or $\mu$ that comes from the $\tau$ decay), and a soft lepton momentum. The fit is performed simultaneously 
on $D^{0}, D^{+}, D^{* 0}$ and $D^{*+}$ and the results are $R(D)=B F(B \rightarrow D \tau v) / B F(B \rightarrow D \ell(e$ or $\mu) v)=0.440 \pm$ $0.058($ stat $) \pm 0.042$ (syst) and $R\left(D^{*}\right)=B F\left(B \rightarrow D^{*} \tau v\right) / B F\left(B \rightarrow D^{*} \ell(e\right.$ or $\left.\mu) v\right)=0.332 \pm 0.024($ stat $) \pm$ 0.018 (syst) ( $B^{0}$ and $B^{+}$isospin constrained results). The measurement of the ratios $R(D)$ and $R\left(D^{*}\right)$ has the advantage that they are experimentally very clean because many systematics cancel in the ratio. Moreover the theoretical predictions in the SM are reliable and affected by a small uncertainties: $R(D)=0.297 \pm 017$ and $R(D)=0.252 \pm 0.003$ [17]. The BaBar result is compatible with the Belle results obtained with a similar technique [18] and exceed the SM prediction by $3.4 \sigma$, if we consider the excess in both the $R^{D}$ and $R^{D^{*}}$ ratios. In the 2HDM of type II, there is a substantial impact on the ratios $R(D)$ and $R\left(D^{*}\right)$ due to the $H^{+}$contribution. Because the preferred $\tan \beta / m_{H^{+}}$regions for $R(D)$ and $R\left(D^{*}\right)$ are not compatible, we are able to put a severe constraint in the $\tan \beta / m_{H^{+}}$plane for the $2 \mathrm{HDM}$ of type II (Fig. 3), that is so disfavoured at more than $3 \sigma$.

The hadronic $B$ tagging technique allows to measure the $B \rightarrow \tau v$ and $B \rightarrow D^{(*)} \tau v$ decays with good precision despite the weak experimental signature. These channels put constraints on $\tan \beta / m_{H^{+}}$ ratio for the $2 \mathrm{HDM}$ that are complementary or stronger than the constraints set by CMS and ATLAS.
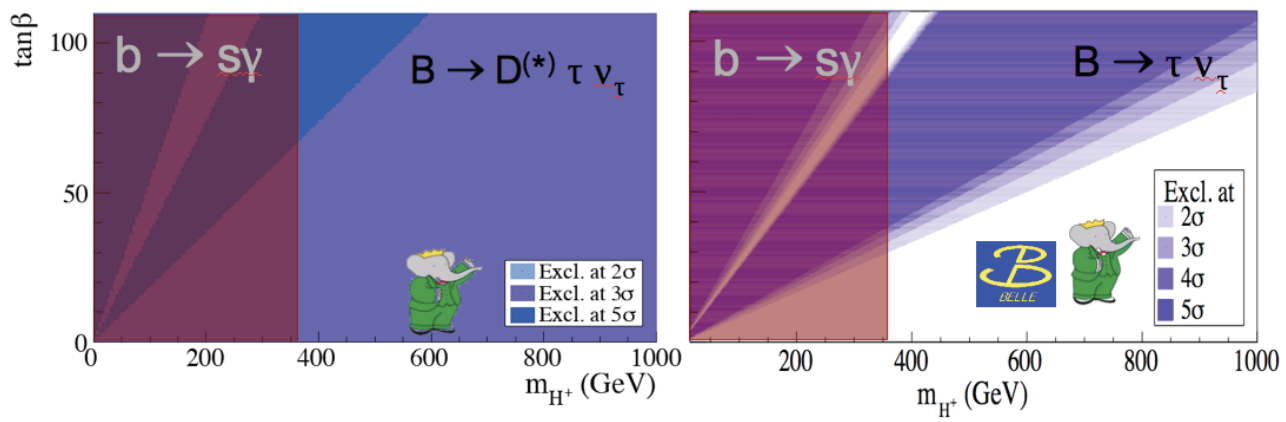

Figure 3. Constraints in the $\tan \beta-m_{H^{+}}$plane in the 2HDM of type II obtained from the BaBar measurement of $R\left(D^{(*)}\right)$ (left) and from the BaBar and Belle results on $B \rightarrow \tau \nu_{\tau}$ branching fraction (right). The vertical red band is the excluded region of $m_{H^{+}}$that comes from $B \rightarrow X_{s} \gamma$ [19]. The $\mathrm{BaBar} R\left(D^{(*)}\right)$ allows to exclude the entire plane at more than $3 \sigma$ level.

\section{Conclusions}

The decays of $B$ mesons with leptons in the final states are a very powerful probe to search for NP effects due to virtual heavy particles in the tree and loop diagrams. Here we presented some recent $\mathrm{BaBar}$ results. NP has not been observed yet, but some interesting tensions are present and these require more studies from both the experimental and theoretical side.

\section{References}

[1] G. Buchalla, A. J. Buras, and M. E. Lautenbacher, Rev. Mod. Phys. 68, 1125 (1996).

[2] J. P. Lees et al. (BaBar Collaboration), Phys. Rev. Lett.112,211802 (2014).

[3] T. Huber, T. Hurt and E. Lunghi, Nucl. Phys. B802,40 (2008).

[4] R. Aaij et al. (LHCb Collaboration), Phys. Rev. Lett. 111,191801 (2013).

[5] R. Gaild, F. Goertz, U. Haisch, JHEP 97, 2014 (2014). 
MESON 2014 $-13^{\text {th }}$ International Workshop on Production, Properties and Interaction of Mesons

[6] A. Atre, T. Han, S. Pascali and B. Zhang, J. High Energy Physics 05, 030 (2009).

[7] S. Davidson, E. Nardi, and Y. Nir, Phys. Rep. 466, 105 (2008).

[8] K. W. Edwards et al. (CLEO Collaboration), Phys. Rev. D 65,111102 (2002).

[9] O. Seon et al. (Belle Collaboration), Phys. Rev. D84,071106 (2011).

[10] J. P. Leeset al. (BaBar Collaboration), Phys. Rev. D 85, 071103 (2012).

[11] R. Aaij et al. (LHCb Collaboration), Phys. Rev. Lett. 108,101601 (2012).

[12] J. P. Leeset al. (BaBar Collaboration), Phys. Rev. D 89, 011102R (2013).

[13] J. P. Leeset al. (BaBar Collaboration), Phys. Rev. D 88, 031102R (2013).

[14] B. Aubert et al. (BaBar Collaboration), Phys. Rev. D 81, 051101(R) (2010); K. Hara et al. (Belle Collaboration), Phys. Rev. D 82, 071101 (2010).

[15] K. Hara et al. (Belle Collaboration), Phys. Rev. Lett. 110, 131801 (2013).

[16] J. P. Lees et al. (BaBar Collaboration), Phys. Rev. Lett 109, 101802 (2012).

[17] S. Fajfer, J. F. Kamenik, and I. Nisandzic, Phys. Rev. D85, 094025 (2012).

[18] I. Adachi et al. (Belle Collaboration), arXiv:0910.4301.

[19] M. Misiak et al. Phys. Rev. Lett. 98022002 (2007). 